\title{
Inhibitory Properties of Nerve-Specific Human Glutamate Dehydrogenase Isozyme by Chloroquine
}

\author{
Myung-Min Choi ${ }^{1}$, Eun-A Kim ${ }^{1,2}$, Soo Young Choi ${ }^{3}$, Tae Ue Kim², Sung-Woo Cho ${ }^{1, *}$ and Seung-Ju Yang,* \\ ${ }^{1}$ Department of Biochemistry and Molecular Biology, University of Ulsan College of Medicine, Seoul 138-736, Korea \\ ${ }^{2}$ Department of Biomedical Laboratory Science, Yonsei University, Wonju 222-701, Korea \\ ${ }^{3}$ Department of Biomedical Sciences, Hallym University, Chunchon 200-702, Korea \\ ${ }^{4}$ Department of Biomedical Laboratory Science, Konyang University, Daejeon 320-718, Korea
}

Received 23 April 2007, Accepted 1 May 2007

\begin{abstract}
Human glutamate dehydrogenase exists in hGDH1 (housekeeping isozyme) and in hGDH2 (nerve-specific isozyme), which differ markedly in their allosteric regulation. In the nervous system, GDH is enriched in astrocytes and is important for recycling glutamate, a major excitatory neurotransmitter during neurotransmission. Chloroquine has been known to be a potent inhibitor of house-keeping GDH1 in permeabilized liver and kidneycortex of rabbit. However, the effects of chloroquine on nerve-specific GDH2 have not been reported yet. In the present study, we have investigated the effects of chloroquine on hGDH2 at various conditions and showed that chloroquine could inhibit the activity of hGDH2 at dose-dependent manner. Studies of the chloroquine inhibition on enzyme activity revealed that hGDH2 was relatively less sensitive to chloroquine inhibition than house-keeping hGDH1. Incubation of hGDH2 was uncompetitive with respect of NADH and non-competitive with respect of 2-oxoglutarate. The inhibitory effect of chloroquine on hGDH2 was abolished, although in part, by the presence of ADP and L-leucine, whereas GTP did not change the sensitivity to chloroquine inhibition. Our results show a possibility that chloroquine may be used in regulating GDH activity and subsequently glutamate concentration in the central nervous system.
\end{abstract}

Keywords: Allosteric regulation, Chloroquine, Glutamate dehydrogenase, Isozymes

Abbreviations: GDH, glutamate dehydrogenase

\footnotetext{
*Co-corresponding authors.

*To whom correspondence should be addressed.

Tel: 82-42-600-6372; Fax: 82-42-543-6370.

E-mail: sjyang@konyang.ac.kr (S.-J. Yang);

swcho@amc.seoul.kr (S.-W. Cho)
}

\section{Introduction}

Schizophrenia is a chronic and disabling brain disorder, which affects up to $1 \%$ of the population worldwide. There is no cure, and treatments, which are mainly based on antagonism of dopamine, serotonin and other neurotransmitter receptors in the brain, are only partially effective (Collier and Li, 2003). Especially, the dysfunction of the glutamatergic system is recognized to take place in the pathogenesis of schizophrenia (Tamminga and Frost, 2001). Smith et al. (2001) have obtained data suggesting that higher expressions of transcripts encoding excitatory amino acid transporters, EAAT1 and EAAT2 were detected in the thalamus of subject with schizophrenia. The evidence which the N-methyl-D-aspartic acid (NMDA) class of glutamate receptor subunits are abnormally expressed in elderly patients with schizophrenia supports glutamate hypothesis of schizophrenia (Dracheva et al., 2001).

Glutamate dehydrogenase (GDH; EC 1.4.1.3) is a family of enzymes catalyzing a reversible deamination of L-glutamate to 2-oxoglutarate directly connected to the Krebs cycle (Fisher, 1985). Recent molecular biological studies have confirmed that two human GDH isozymes (hGDH1 and hGDH2) of distinct genetic origin are expressed in human tissues. (Hussain et al., 1989; Shashidharan et al., 1997; Zaganas et al., 2002; Plaitakis et al., 2003). hGDH1 (housekeeping GDH) is expressed widely, whereas hGDH2 (nerve-specific GDH) is specific for neural and testicular tissues (Hanauer et al., 1987; Mavrothalassitis et al., 1988; Anagnou et al., 1993; Michaelidis et al., 1993; Shashidharan et al., 1994). In brain, hGDH may have a dual function that involves glutamate neurotransmitter availability and energy metabolism. However, the functional significance and reaction mechanism of nerve-specific GDH isozyme in nerve tissue remains to be studied.

Chloroquine is a drug with safe clinical use in the treatment of malaria and inflammatory disorders. Also, it has found 
utility in the treatment of rheumatic disorders such as lupus erythematosus and rheumatoid arthritis (Tett et al., 1990). In addition, there are some reports that hydroxychloroquine affects the synthesis and secretion of proinflammatory cytokines such as interleukin (IL)-1- $\alpha$, IL-6, tumor necrosis factor- $\alpha$ (TNF- $\alpha)$, and interferon- $\gamma$ (Sperber et al., 1993; Van den Boren et al., 1997) and this molecule may affect neuronal functioning and psychiatric symptoms (Nawa et al., 1997; Reichenberg et al., 2001). Despite of some reports about abnormality of neuron such as death of cerebellar granule neurons and accumulation of GM1 ganglioside in Rab5positive enlarged early endosomes by chloroquine (Shacka et al., 2006, Yuyama et al., 2006), hydroxychloroquine treatment in patients with schizophrenia was associated with a greater probability of release from the hospital and with improved psychiatric symptoms and chloroquine can depress in vitro neuronal activity, perhaps through inhibition of membrane calcium channels (Desta et al., 2002; O'Shaughnessy et al., 2003). In this study, we investigated whether chloroquine is also potent inhibitor of hGDH2 and is able to be a candidate for treatment of schizophrenia.

\section{Materials and Methods}

Materials. NADH, $\mathrm{NAD}^{+}$, 2-oxoglutarate, $\mathrm{ADP}$, and factor $\mathrm{Xa}$ were purchased from Sigma Chemical Co. Previously constructed synthetic hGDH2 gene was used (Cho et al., 2001). Pre-cast gels for sodium dodecyl sulfate-polyacrylamide gel electrophoresis and pre-stained marker proteins for Western blot were purchased from Novex. Low molecular weight marker proteins were purchased from Bio-Rad. All other chemicals and solvents were reagent grade or better.

Bacterial strains. E. coli DH5 $\alpha$ (Hanahan, 1983) was purchased from Invitrogen and used as the host strain for plasmid-mediated transformations for cassette mutagenesis. E. coli PA340 (thr-1 fhuA2 leuB6 lacY1 supE44 gal-6 gdh-1 hisG1 rfbD1 galP63 v(gltBF) 500 rpsL19 malT1 xyl-7 mtl-2 argH1 thi-1; kindly provided by Dr. Mary K. B. Berlyn, E. coli Genetic Stock Center, Yale University, New Haven, CT, USA) lacked both GDH and glutamate synthase activities (Teller et al., 1992) and was used to test plasmids for GDH activity. E. coli BL21(DE3) (Studier et al., 1986) was used for high-level expression of the recombinant human GDH2.

Construction and expression of synthetic hGDH2. The design of the synthetic $h G D H 2$ gene was based on the amino acid sequence of the mature hGDH2 (Shashidharan et al., 1994). The synthetic $h G D H 2$ gene was constructed from the $h G D H 1$ gene previously synthesized (Yang et al., 2003) by cassette mutagenesis at different amino acid residues not common between the GDH1 and GDH2 isozymes (Shashidharan et al., 1994). hGDH2 proteins were expressed in E. coli strain BL21(DE3). The gene expression level in the crude extract was examined by Western blot using monoclonal antibodies against recombinant hGDH. The expressed proteins were purified to homogeneity as described before (Cho et al., 2001) and analyzed by SDS-PAGE. To remove five additional N-terminal residues (M-I-E-G-R), the purified hGDH2 was treated with factor $\mathrm{Xa}(10 \mu \mathrm{g} / \mathrm{mg}$ GDH) in a reaction mixture of $20 \mathrm{mM}$ Tris- $\mathrm{HCl} / 100$ $\mathrm{mM} \mathrm{NaCl} / 2 \mathrm{mM} \mathrm{CaCl}$. After incubation at room temperature for $3 \mathrm{~h}$, the reaction mixtures were purified by a high performance liquid chromatography (HPLC) Protein-Pak 300SW gel filtration column as described before (Cho et al., 2001). The purified protein was analyzed by SDS-PAGE and Western blot analysis (Kim et al., 2006). HPLC-purified hGDH 2 was subjected to automated amino acid sequencing. Steady-state kinetic parameters were determined with the purified proteins unless otherwise indicated.

Enzyme kinetics. hGDH activity was measured spectrophotometrically in the direction of reductive amination of 2-oxoglutarate by following the decrease in absorbance at $340 \mathrm{~nm}$ as described before (Cho et al., 1995). All assays were performed in duplicate and initial velocity data were correlated with a standard assay mixture containing $50 \mathrm{mM}$ triethanolamine, $\mathrm{pH} 8.0,100 \mathrm{mM}$ ammonium acetate, $0.1 \mathrm{mM} \mathrm{NADH}$, and $2.6 \mathrm{mM}$ EDTA at $25^{\circ} \mathrm{C}$. The reaction started with the addition of 2-oxoglutarate to $10 \mathrm{mM}$ final concentration. Since $E$. coli only has an NADP(H)-dependent GDH (Hanahan, 1983), the enzyme assay was performed with NADH as a coenzyme as described elsewhere (Cho et al., 1995). One unit of enzyme was defined as the amount of enzyme required for oxidizing $1 \mu \mathrm{mol}$ of $\mathrm{NADH} / \mathrm{min}$ at $25^{\circ} \mathrm{C}$.

For determination of $K_{m}$ and $V_{\text {max }}$ values, the assays were carried out by varying the substrate under investigation while keeping the other substrate and reagents at the optimal concentrations. $K_{i}$ values of hGDH2 were obtained in the presence of $200 \mu \mathrm{M}$ chloroguine from the initial velocity data and linear regression analysis of double-reciprocal plots as described elsewhere (Yang et al., 2004). GDH activity was measured in the presence of $1 \mathrm{mM}$ ADP. The data represent the mean of two independent experiments. Duplicate of the kinetic values differed less than $5 \%$.

Statistical analysis. Unless otherwise mentioned, each experimental point represents the mean of triplicate determinations from different preparations and standard deviation is indicated as error bar in each figure. At some points, error bars were omitted in the figures for clarity purpose.

\section{Results and Discussion}

Chloroquine has been known to be a potent inhibitor of house-keeping GDH in permeabilized liver and kidney-cortex of rabbit (Jarzyna et al., 1997). The effects of chloroquine on house-keeping human GDH1 (Fig. 1) was consistent with those of liver and kidney enzymes (Jarzyna et al., 1997). However, the effects of chloroquine on nerve-specific hGDH2 have not been reported yet. In the present study, we have investigated the effects of chloroquine on hGDH2 at various conditions and showed that chloroquine can inhibit the activity of hGDH2 at dose-dependent manner. Studies of the chloroquine inhibition on enzyme activity revealed that hGDH2 was relatively less sensitive to chloroquine inhibition than house-keeping GDH1 (Fig. 1). The $\mathrm{IC}_{50}$ values were 


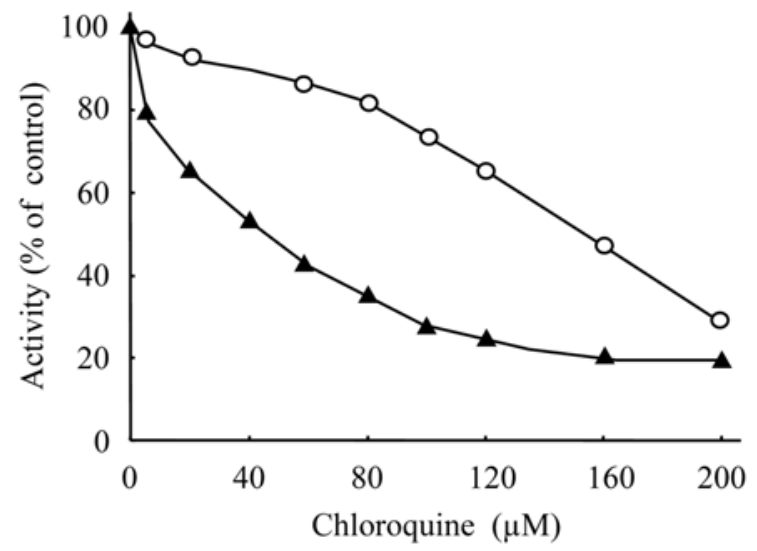

Fig. 1. Concentration-dependent inhibition of hGDH1 and hGDH2 by chloroquine. Data points represent mean values from at least two experimental determinations and are expressed as percentage of each control. Duplicate of the kinetic values differed less than 5\%.

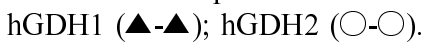

$50 \mu \mathrm{M}$ and $140 \mu \mathrm{M}$ for hGDH1 and hGDH2, respectively. We have applied the $200 \mu \mathrm{M}$ concentration of chloroquine for the rest of studies. When the inhibitory effects were examined with various concentrations of 2-oxoglutarate at a fixed concentration of NADH, it showed a non-competitive inhibition against 2-oxoglutarate with a $K_{i}$ value of $56 \mu \mathrm{M}$ (Fig. 2A). In contrast, it showed an uncompetitive inhibition against NADH with a $K_{i}$ value of $373 \mu \mathrm{M}$ (Fig. 2B). These results indicate that the binding site of chloroquine is different from those of 2-oxoglutarate and NADH and the inhibitory effects may be due to the conformational change caused by the binding of the drug.

Since it is well documented that GDH is highly activated by allosteric activators such as ADP, L-leucine, and GTP (Fisher et al., 1985; Cho et al., 1995), it is important to take account of the regulators when considering the inhibitory actions of drugs on GDH. Therefore, we have examined the effects of ADP, L-leucine, and GTP on the inhibition by chloroquine. Study of the ADP stimulatory curves revealed that ADP induced a concentration dependent activation of hGDH2 (Fig. 3). The inhibitory effect of chloroquine on hGDH2 was abolished, although in part, by the presence of ADP up to $0.5 \mathrm{mM}$ (Fig. 3). More profound protection of hGDH2 against chloroquine inhibition was observed with Lleucine, another well known allosteric activator of GDH. As shown in Fig. 4, more than $80 \%$ of hGDH2 activity was recovered in the presence of $10 \mathrm{mM}$ L-leucine. Previously, it was reported that ADP and L-leucine did not affect the inhibitory actions of chloroquine in house-keeping liver and renal mitochondria GDH (Jarzyna et al., 1997). The discrepancies between the effects of chloroquine on housekeeping GDH1 and nerve-specific GDH2 indicate that the mechanisms of action of chloroquine on GDH1 and GDH2 may differ from each other as well as the different regulatory mechanism of ADP and L-leucine on these GDH isozymes.
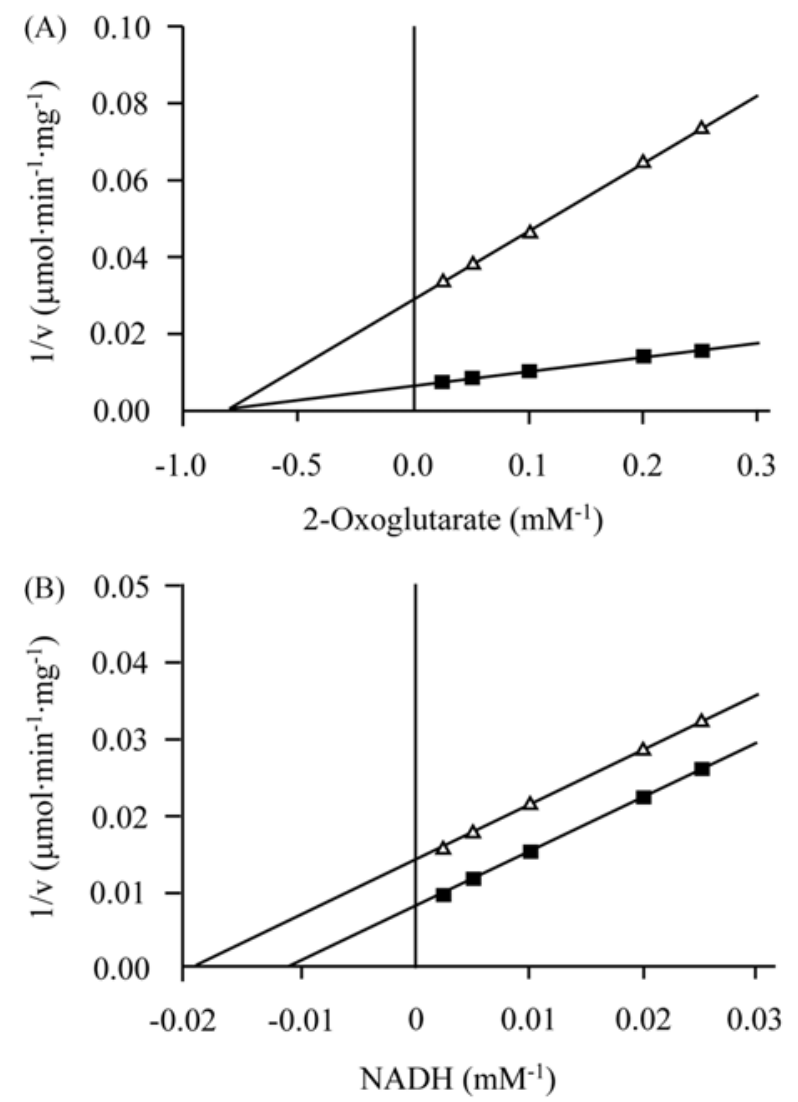

Fig. 2. Inhibition of hGDH2 by chloroquine. $K_{m}, K_{i}$, and $V_{\max }$ values of hGDH2 were obtained in the presence and absence of $200 \mu \mathrm{M}$ chloroguine from the initial velocity data and linear regression analysis of double-reciprocal plots as described in Materials and Methods. hGDH2 activity was measured in the presence of $1 \mathrm{mM}$ ADP. The data represent the mean of two independent experiments. Duplicate of the kinetic values differed less than 5\%. $\mathbf{\square}$, control (hGDH2 only); $\Delta$ hGDH2 with $200 \mu \mathrm{M}$ chloroquine.

For instance, there are differences in the sensitivity to ADP and L-leucine between hGDH1 and hGDH2 (Plaitakis et al. 2000, 2003; Yang et al., 2004).

We also have investigated the effects of GTP, an allosteric inhibitor, on chloroquine inhibition of hGDH2. Interestingly, unlike to the results in ADP or L-leucine activation studies, the changes in the sensitivity to chloroquine inhibition were not observed in the presence of GTP (Fig. 5) Unlike to hGDH1, hGDH2 has been known to be resistant to GTP inhibition (Zaganas and Plaitakis, 2003). As GTP levels are generally higher in brain than in other tissues, GTP may not be a suitable modulator for hGDH2 activity in the nerve tissue. Taken together with the dependency on available ADP levels, it will permit the recruitment of the enzyme under conditions of low energy charge (high ADP/ATP ratio), such as those occurring under intense glutamatergic neurotransmission. Therefore, hGDH2 may have evolved to function in the particular environment that prevails in glial cells following glutamate uptake. 


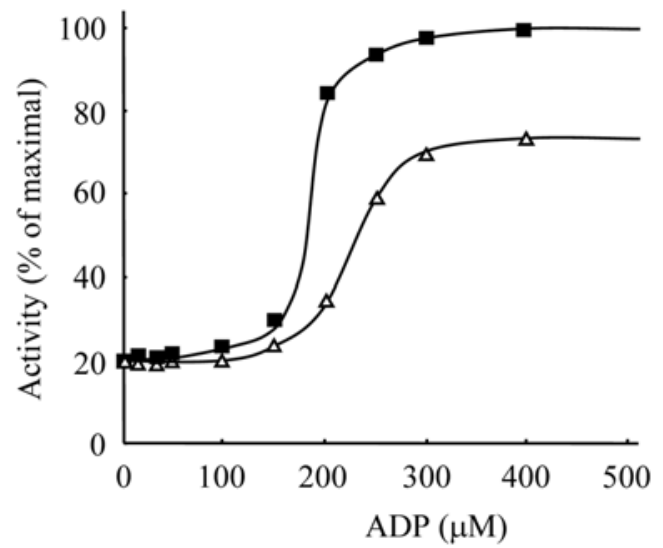

Fig. 3. Effects of ADP on inhibition of hGDH2 by chloroquine. Data points represent mean values from at least two experimental determinations and are expressed as percentage of maximal activity. GDH activity was measured in the direction of reductive amination of 2-oxoglutarate with $\mathrm{NADH}$ in standard assay mixture at $\mathrm{pH} 8.0$ in the presence of increasing concentrations of ADP. All assays were performed with purified samples. $\boldsymbol{\square}$, control (hGDH2 only); $\Delta$ hGDH2 with $200 \mu \mathrm{M}$ chloroquine.

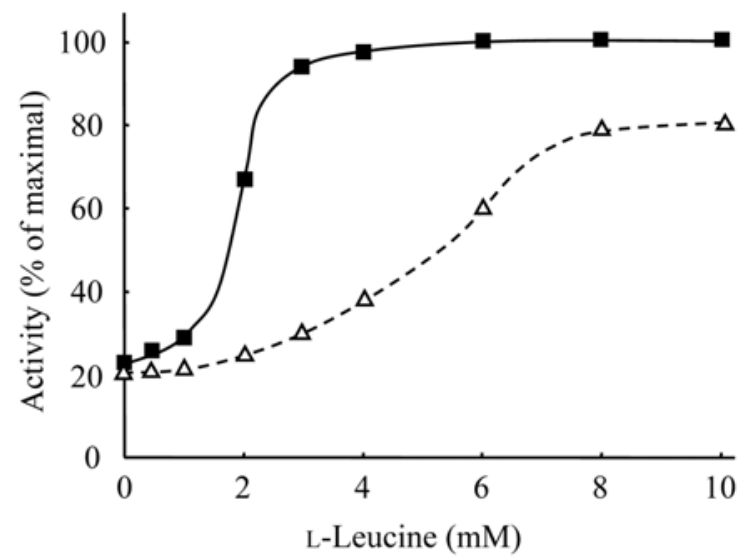

Fig. 4. Effects of L-leucine on inhibition of hGDH2 by chloroquine. Data points represent mean values from at least two experimental determinations and are expressed as percentage of maximal activity. GDH activity was measured in the direction of reductive amination of 2-oxoglutarate with NADH in standard assay mixture at $\mathrm{pH} 8.0$ in the presence of increasing concentrations of L-leucine. All assays were performed with purified samples. control (hGDH2 only); $\Delta$ hGDH2 with $200 \mu \mathrm{M}$ chloroquine.

The glutamatergic hypothesis of schizophrenia was based primarily on data showing changes in glutamate levels in brain and cerebrospinal liquid (Tsai et al., 1995). Previously, Shashidharan et al. (1994) reported the presence of two human GDH-specific genes encoding highly homologous polypeptides. The first gene is expressed in all tissues, designated GLUDI (housekeeping GDH), whereas the second gene is expressed specifically in neural and testicular tissues,

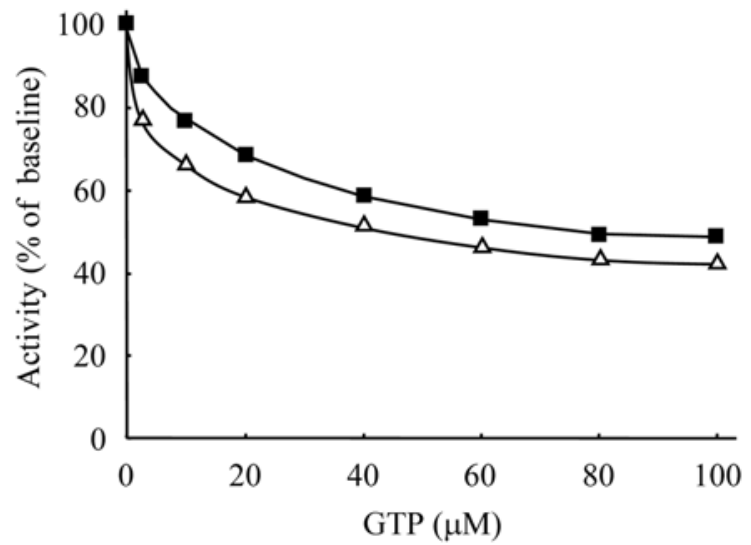

Fig. 5. Effects of GTP on inhibition of hGDH2 by chloroquine. Data points represent mean values from at least two experimental determinations and are expressed as percentage of baseline activity (no GTP added). GDH activity was measured in the direction of reductive amination of 2-oxoglutarate with NADH in standard assay mixture at $\mathrm{pH} 8.0$ in the presence of increasing concentrations of GTP. All assays were performed with purified samples and in the presence of $1 \mathrm{mM}$ ADP. $\mathbf{\square}$, control (hGDH2 only); $\Delta \mathrm{hGDH} 2$ with $200 \mu \mathrm{M}$ chloroquine.

designated GLUDI (nerve tissue-specific GDH). Very recently, it also has been reported that nerve tissue-specific and housekeeping human GDHs are regulated by distinct allosteric mechanism (Shashidharan et al., 1997; Plaitakis et al., 2000). Immunoreactivities of all three GDH isoenzymes are significantly elevate in prefrontal cortex of patients with schizophrenia compared to controls (Burbaeva et al., 2003). Except for GDH3, the isoform partially associated with particulate (membrane) fraction, significantly changed levels of immunoreactivities of GDH2 was higher than GDH1 (Burbaeva et al., 2003). Therefore, GDH2 inhibition by specific inhibitor can regulate glutamate level in neuronal or glial systems and specific GDH2 inhibitor can be a drug of antipsychotic diseases, such as schizophrenia. There are some reports that chloroquine can depress in vitro neuronal activity, perhaps through inhibition of membrane calcium channels (O'Shaughnessy et al., 2003). It also has been reported that hydroxychloroquine is associated with probability of release from the hospital and with improved psychiatric symptoms (Desta et al., 2002). In the present study, we have shown that chloroquine is a potent inhibitor for the nerve-specific hGDH2 and supported the possibility that chloroquine may be one of the candidates for the anti-schizophrenic drugs. The effects of chloroquine on GDH activity in vivo remain to be elucidated and are in progress in our laboratory.

Acknowledgments This work was supported by grants from the Korea Research Foundation (KRF-2006-005-J00802) and from the Korea Science and Engineering Foundation (R012002-000-00125-0). 


\section{References}

Anagnou, N. P., Seuanez, H., Modi, W., O’Brien, S. J., Papamatheakis, J. and Moschonas, N. K. (1993) Chromosomal mapping of two members of the human glutamate dehydrogenase (GLUD) gene family to chromosomes 10q22.3-q23 and Xq22-q23. Hum. Hered. 43, 351-356.

Burbaeva, G. S., Boksha, I. S., Turishcheva, M. S., Vorrobyeva, E. A., Savushkina, O. K. and Tereshkina, E. B., (2003) Glutamine synthetase and glutamate dehydrogenase in the prefrontal cortex of patients with schizophrenia. Prog. Neuro-Psychoph. 27, 675680

Cho, S.-W., Lee, J. and Choi, S. Y. (1995) Two soluble forms of glutamate dehydrogenase isoproteins from bovine brain. Eur. $J$. Biochem. 233, 340-346.

Cho, S.-W., Yoon, H.-Y., Ahn, J.-Y., Lee, E.-Y. and Lee, J. (2001) Cassette mutagenesis of lysine 130 of human glutamate dehydrogenase: An essential residue in catalyis. Eur. J. Biochem. 268, 3205-3213.

Collier, D. A. and Li, T., (2003) The genetics of schizophrenia: glutamate not dopamine? Eur. J. Pharmacol. 480, 177-184.

Desta, M., Tadesse, A., Gebre, N., Barci., B. M., Torrey, E. F. and Bnable, M. B. (2002) Controlled trial of hydroxychloroquine in schizophrenia. J. Clin. Psychopharm. 22, 507-510.

Dracheva. S., Marras, S. A. E., Elhakem, S. L., Kramer, F. R., Davis, K. L. and Haroutunian, V. (2001) N-Methyl-D-aspartic acid receptor expression in the dorsolateral prefrontal cortex of elderly patients with schizophrenia. Am. J. Psychiat. 158, 1400-1410.

Fisher, H.F . (1985) L-Glutamate dehydrogenase from bovine liver. Methods Enzymol. 113, 16-27.

Hanahan, D. (1983) Studies on transformation of Escherichia coli with plasmids. J. Mol. Biol. 166, 557-580.

Hanauer, A., Mattei, M. G. and Mandel, J. L. (1987) Presence of a TaqI polymorphism in the human glutamate dehydrogenase (GLUD) gene on chromosome 10. Nucleic Acids Res. 15, 6308.

Hussain, M. H., Zannis, V. I. and Plaitakis, A. (1989) Characterization of glutamate dehydrogenase isoproteins purified from the cerebellum of normal subjects and patients with degenerative neurological disorders and from human neoplastic cell lines. $J$. Biol. Chem. 264, 20730-20735.

Jarzyna, R., Lenarcik, E. and Bryla, J. (1997) Chloroquine is a potent inhibitor of glutamate dehydrogenase in liver and kidney-cortex of rabbit. Pharmacol. Res. 35, 79-84.

Kim, S. Y., An, J. J., Kim, D. W., Choi, S. H., Lee, S. H., Hwang, S.I., Kwon, O.-S., Kang, T.-C., Won, M. H., Cho, S.-W., Park, J., Eum, W. S., Lee, K. S. and Soo Young Choi (2006) Tat-mediated protein transduction of human brain pyridoxine-5-P oxidase into PC12 cells. J. Biochem. Mol. Biol. 39, 76-83.

Mavrothalassitis, G., Tzimagiorgis, G., Mitsialis, A., Zannis, V. I., Plaitakis, A., Papamatheakis, J. and Moschonas, N. K. (1988) Isolation and characterization of cDNA clones encoding human liver glutamate dehydrogenase: evidence for a small gene family. Proc. Natl. Acad. Sci. USA 85, 3494-3498.

Michaelidis, T. M., Tzimagiorgis, G., Moschonas, N. K. and Papamatheakis, J. (1993) The Human Glutamate Dehydrogenase Gene Family: Gene Organization and Structural Characterization. Genomics 16, 150-160.

Nawa, H., Takahashi, M. and Patterson, P. H. (2000) Cytokine and growth factor involvement in schizophrenia: support for the developmental model. Mol. Psychiatry 5, 594-603.
O'Shaughnessy, T. J., Zim, B., Ma, W., Shaffer, K. M., Stenger, D. A., Zamani, K., Gross, G. W. and Pancrazio, J. J., (2003) Acute neuropaharmacologic action of chloroquine on cortical neurons in vitro. Brain Res. 959, 280-286.

Plaitakis, A., Spanaki, C., Mastorodemos, V. and Zaganas, I. (2003) Study of structure-function relationships in human glutamate dehydrogenases reveals novel molecular mechanisms for the regulation of the nerve tissue-specific (GLUD2) isoenzyme. Neurochem. Int. 43, 401-410.

Plaitakis, A., Metaxari. M. and Shashidharan, P., (2000) Nerve tissue-specific (GLUD2) and housekeeping (GLUD2) and housekeeping (GLUD1) human glutamate dehydrogenases are regulated by distinct allosteric mechanisms: Implications for biological function. J. Neurochem. 75, 1862-1869.

Reichenberg, A., Yirmiya, R. and Schuld, A. (2001) Cytokineassociated emotional and cognitive disturbances in humans. Arch Gen Psychiatry, 58 445-452.

Shacka, J. J., Klocke, B. J., Shibata, M., Uchiyama, Y., Datta, G., Schmidt, R. E. and Roth, K. A. (2006) Bafilomycin A1 inhibits chloroquine-induced death of cerebellar granule neurons. Mol. Pharmacol. 69, 1125-1136.

Shashidharan, P., Michaelidis, T. M., Robakis, N. K., Kresovali, A., Papamatheakis, J. and Pliatakis, A. (1994) Novel human glutamate dehydrogenase expressed in neural and testicular tissues and encoded by an X-linked intronless gene. J. Biol. Chem. 269, 16971-16976.

Shashidharan, P., Clarke, D. D., Ahmed, N., Moschonas, N. and Plaitakis, A. (1997) Nerve tissue-specific human glutamate dehydrogenase that is thermolabile and highly regulated by ADP. J. Neurochem. 68, 1804-1811.

Smith, R. E., Haroutunian, V., Davis, K. L. and Meador-Woodruff, J. H. (2001) Expression of excitatory amino acid transporter transcripts in the thalamus of subjects with schizophrenia. Am. J. Psychiatry. 158, 1393-1399.

Sperber, K., Quraishi, H. and Kalb T. H. (1993) Selective regulation of cytokine secretion by hydroxychloroquine: inhibition of interleukin 1 alpha and IL-6 in human monocytes and T cells. $J$. Rheumatol. 20, 803-808.

Studier, F. W. and Moffatt, B. A. (1986) Use of bacteriophage T7 RNA polymerase to direct selective high-level expression of cloned genes. J. Mol. Biol. 189, 113-130.

Tamminga, C. A. and Frost, D. O., (2001) Changing concepts in the neurochemistry of schizophrenia. Am. J. Psyciat. 158, 1365-1366.

Teller, J. K., Smith, R. J., McPherson, M. J., Engel, P. C. and Guest, J. R. (1992) Correlation of intron-exon organization with the three-dimensional structure in glutamate dehydrogenase. Eur. $J$. Biochem. 206, 151-159.

Tett, S., Cutler, D., and Day, R. (1990) Antimalarials in rheumatic diseases. Bailieres Clin. Rheumatol. 4, 467-489

Tsai, G., Passani, L. A., Slusher, B. S., Baer, L., Kleinman, J. E. and Coyle, J. T. (1995) Abnormal excitatory neurotransmitter metabolism in schizophrenic brain. Arch. Gen. Psychiatry 52, 829-836.

Van den Boren, B. E., Dijkmans, B. A. and de Rooij, H. H. (1997) Chloroquine and hydroxychloroquine equally affect tumor necrosis factor-alpha, interleukin 6, and interferon-gamma production by peripheral blood mononuclear cells. J. Rheumatol, 24, 55-60.

Yang, S.-J., Huh, J.-W., Kim, M. J., Lee, W.-J., Kim, T. U., Choi, S. Y. and Cho, S.-W. (2003) Regulatory effects of 5'-deoxypyridoxal 
on glutamate dehydrogenase activity and insulin secretion in pancreatic islets. Biochimie 85, 581-586.

Yang, S.-J., Huh, J.-W., Hong, H.-N., Kim, T. U. and Cho, S.-W. (2004) Important role of Ser443 in different thermal stability of human glutamate dehydrogenase isozymes. FEBS Lett. 562, 5964.

Yuyama, K., Yamamoto, N. and Yanagisawa, K. (2006) Chloroquineinduced endocytic pathway abnormalities: Cellular model of
GM1 ganglioside-induced Ab fibrillogenesis in Alzheimer's disease. FEBS Lett. 580, 6972-6976.

Zaganas, I. and Plaitakis, A. (2002) Single amino acid substitution (G456A) in the vicinity of the GTP binding domain of human housekeeping glutamate dehydrogenase markedly attenuates GTP inhibition and abolishes the cooperative behavior of the enzyme. J. Biol. Chem. 277, 26422-26428. 